\title{
Planning Of Human Resource Competency Development In PT.XYZ With Taguchi Method
}

\author{
M. Kertaningtyas ${ }^{1}$; D.W.L. Basuki ${ }^{2}$ \\ ${ }^{1,2}$ Industrial Engineering Faculty, National of Technology Institute Malang \\ Email : mariza@fti.itn.ac.id
}

\begin{abstract}
The problem of human resources is still a concern within the company to remain competitive in this globalization world. This shows that the problem of human resources greatly affect the implementation and success of the company in achieving goals and objectives. The company demand to obtain the development process and get quality human resources more urgent. And the development of human resource competence is necessary. This study uses experimental testing with several parameters of validity and reliability testing. For testing analysis using Taguchi Method. Based on the Response Table for Signal to Noise Ratios Nominal is best obtained taguchi test results obtained values obtained from the effect plot for means with the approach of table of means, then the intellectual competence is needed for the improvement of HR performance..
\end{abstract}

Keywords HR, Competence, Taguchi Method

Paper type Research paper

\section{INTRODUCTION (HEADING 1)}

Human resources are currently in the spotlight for companies to survive in this era of globalization. Although it has been supported with the best facilities and infrastructure, HR still becomes the key point in determining the success of the company [1]. The demand to obtain maximum results, by developing and maintaining quality human resources increasingly urgent.

The necessary changes to support top management as the first step in improving the role of the human resources fungus, to support the success of an organization [2]. Organizational goals will be achieved with positive performance and good work ethic. Vice versa, the organization will experience obstacles if the performance of human resources decreased even not effective [3]. Effort to get performance from good human resources, hence competence test is needed based on certain time and size.

Creation of qualified human resources has a big role to determine the success or failure in organizing various activities to achieve company goals. Assessment of employee performance as an agent in the organization should be projected into the HR performance standard in accordance with its work unit. It aims to improve the process of HR performance [4].

Assessment indicators in a company include the work, the ability of employees, customer service and employee performance improvement. Individually can be classified through intellectual, emotional, and social competence.

The Taguchi method in an experiment of fundamental things that needs to be defined is the purpose of doing experiments so that later after the experiment can be analyzed against the output results to meet the initial goal. In this research, the experiment is done by taguchi method, to get optimum result [9]. Therefore, in this research the experiment is used to know the combination of the level of each optimum factor and produce the highest level of interest and understanding. The purpose of this study, to develop the competenc [7].

\section{METHOD}

Research conducted using direct research method (experimental research) by involving several variables, namely knowledge, skill, attitude, and work effectiveness. This research uses the method of designing tolerance in the optimization of human resources. Technique Data analysis in this study using ANOVA to determine the significance and the influence of factors, and orthogonal arrays Taguchi so that the design used in the experiment more efficient, and data analysis.

Research variable

a. $\quad$ Fixed Variables: Intellectual, Emotional, and Social Competencies.

b. Free Variable: K (understanding theory, rules, possess knowledge, creative idea)

$\mathrm{E}$ (timely, good quality, SOP, attendance) 
S (Social norms, cooperation with rules and friends, and communication)
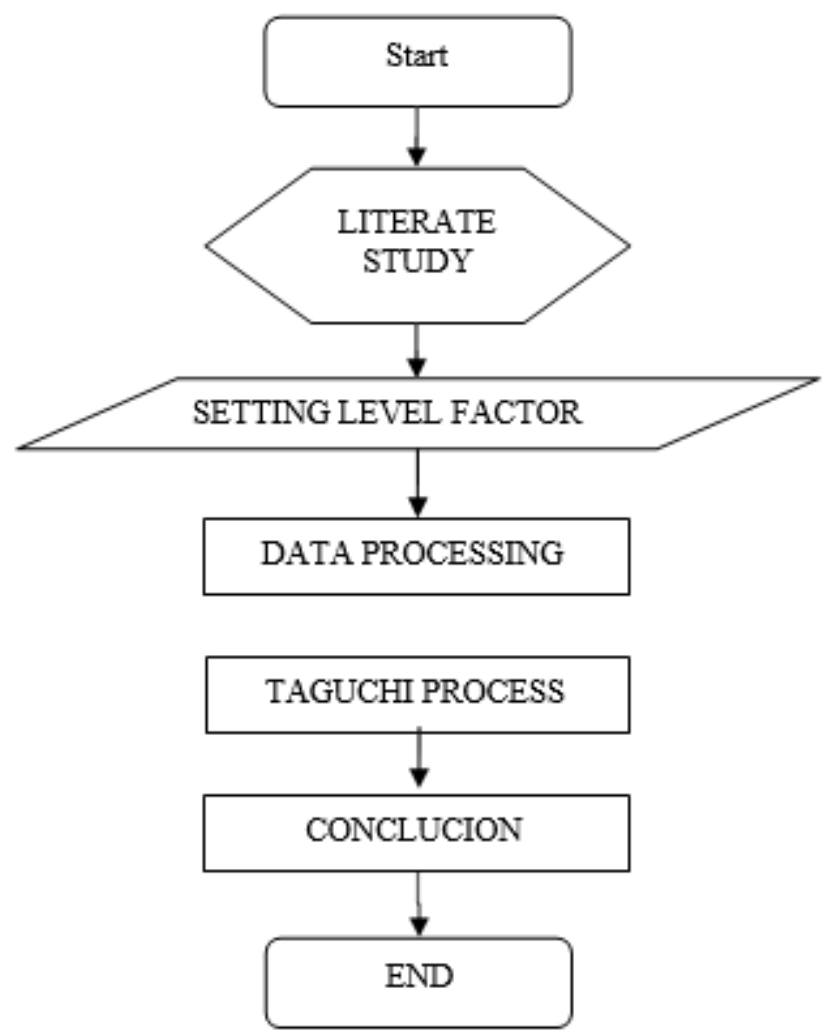

Fig. 1. Flow Chart of Research

Research Process

1. Literature Study, aims to obtain the literature needed in the determination of the next step,

2. Field study, in the form of survey location to get situation problem existing in company,

3. Problem formulation, this is developed from research variables

4. Data retrieval,

5. Data Processing, Analisis,

Table 1. Taguchi Setting Level Factor

\begin{tabular}{|c|c|c|c|c|}
\hline \multicolumn{5}{|c|}{ SETTING LEVEL } \\
\hline FACTOR & I & II & III & IV \\
\hline $\begin{array}{l}\text { INTELLECTUAL } \\
\text { COMPETENCY }\end{array}$ & $\begin{array}{c}\text { K1 } \\
\text { UNDERSTAND } \\
\text { THE THEORY }\end{array}$ & $\begin{array}{c}\text { K2 } \\
\text { UNDERSTANDING } \\
\text { RULE }\end{array}$ & $\begin{array}{c}\text { K3 } \\
\text { HAVE } \\
\text { KNOWLEDGE }\end{array}$ & \begin{tabular}{l}
\multicolumn{1}{c}{ K4 } \\
HAVE A \\
CREATIVE \\
$\quad$ IDEAS
\end{tabular} \\
\hline $\begin{array}{l}\text { EMOTIONAL } \\
\text { COMPETENCY }\end{array}$ & $\begin{array}{c}\text { E1 } \\
\text { IMPLEMENTING } \\
\text { TIMELY WORK }\end{array}$ & $\begin{array}{c}\text { E2 } \\
\text { IMPLEMENT } \\
\text { WORK WITH } \\
\text { GOOD QUALITY }\end{array}$ & $\begin{array}{c}\text { E3 } \\
\text { COMPLETING } \\
\text { THE JOB } \\
\text { ACCORDING TO } \\
\text { THE } \\
\text { PROCEDURES }\end{array}$ & $\begin{array}{c}\text { E4 } \\
\text { PRESENT ON } \\
\text { THE TIME }\end{array}$ \\
\hline $\begin{array}{c}\text { SOCIAL } \\
\text { COMPETENCY }\end{array}$ & $\begin{array}{c}\text { S1 } \\
\text { COMPLYING } \\
\text { SOCIAL NORMA }\end{array}$ & \begin{tabular}{l}
\multicolumn{1}{c|}{ S2 } \\
COOPERATE WITH \\
RULES
\end{tabular} & $\begin{array}{c}\text { S3 } \\
\text { COOPERATION } \\
\text { WORKING FOR } \\
\text { WORKERS }\end{array}$ & $\begin{array}{c}\text { S4 } \\
\text { COMMUNICATE } \\
\text { INTER FRIENDS } \\
\text { WELL }\end{array}$ \\
\hline
\end{tabular}




\section{DISCUSSION}

Table 2. Taguchi Method test

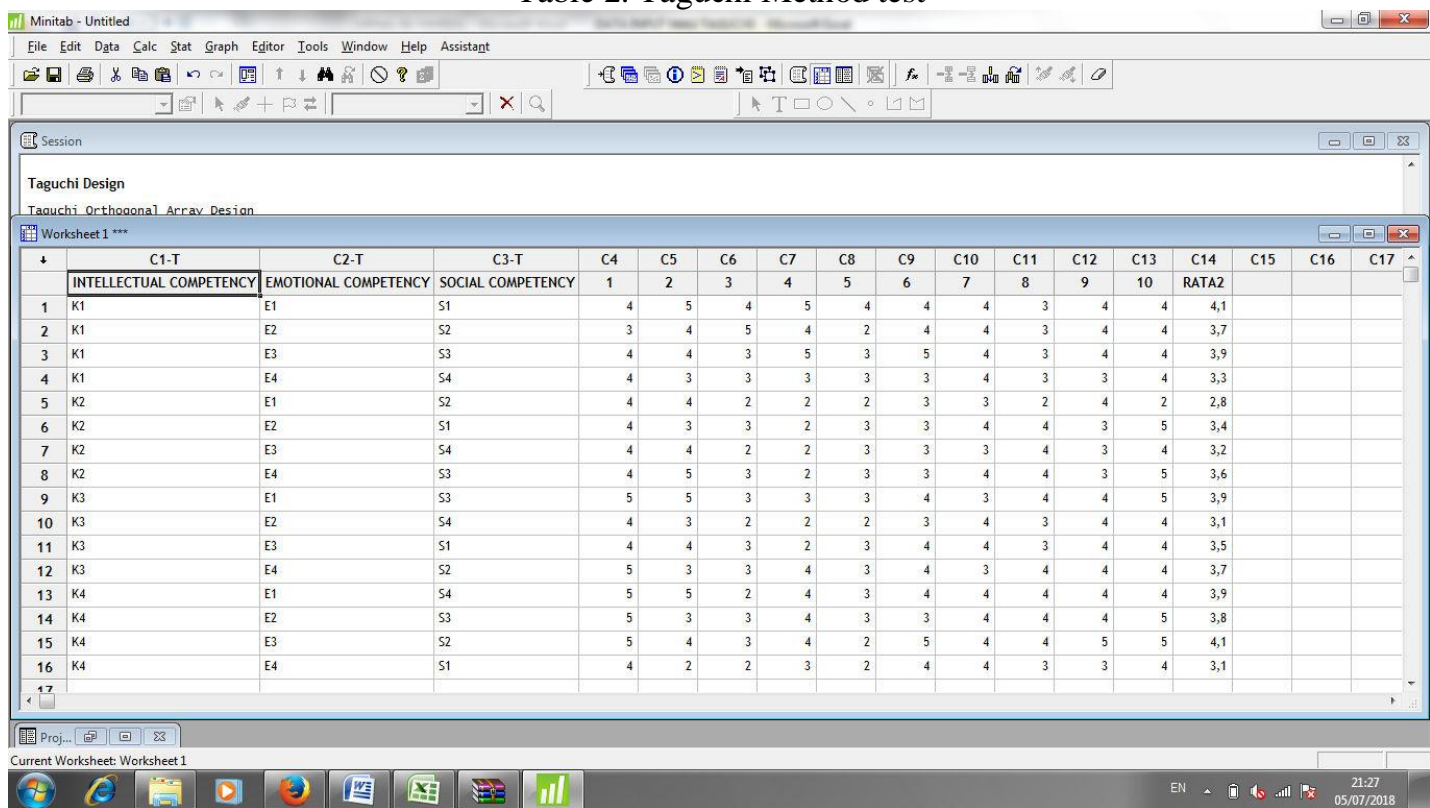

Table.3 Test Resukt

\begin{tabular}{|c|c|c|c|c|c|c|c|c|c|c|c|c|}
\hline \multirow{2}{*}{$\begin{array}{l}\text { INTELLECTUAL } \\
\text { COMPETENCY }\end{array}$} & \multirow{2}{*}{$\begin{array}{l}\text { EMOTIONAL } \\
\text { COMPETENCY }\end{array}$} & \multirow{2}{*}{$\begin{array}{c}\text { SOCIAL } \\
\text { COMPETENCY }\end{array}$} & \multicolumn{10}{|c|}{ Responden } \\
\hline & & & 1 & 2 & 3 & 4 & 5 & 6 & 7 & 8 & 9 & 10 \\
\hline 1 & 1 & 1 & 4 & 5 & 4 & 5 & 4 & 4 & 4 & 3 & 4 & 4 \\
\hline 1 & 2 & 2 & 3 & 4 & 5 & 4 & 2 & 4 & 4 & 3 & 4 & 4 \\
\hline 1 & 3 & 3 & 4 & 4 & 3 & 5 & 3 & 5 & 4 & 3 & 4 & 4 \\
\hline 1 & 4 & 4 & 4 & 3 & 3 & 3 & 3 & 3 & 4 & 3 & 3 & 4 \\
\hline 2 & 1 & 2 & 4 & 4 & 2 & 2 & 2 & 3 & 3 & 2 & 4 & 2 \\
\hline 2 & 2 & 1 & 4 & 3 & 3 & 2 & 3 & 3 & 4 & 4 & 3 & 5 \\
\hline 2 & 3 & 4 & 4 & 4 & 2 & 2 & 3 & 3 & 3 & 4 & 3 & 4 \\
\hline 2 & 4 & 3 & 4 & 5 & 3 & 2 & 3 & 3 & 4 & 4 & 3 & 5 \\
\hline 3 & 1 & 3 & 5 & 5 & 3 & 3 & 3 & 4 & 3 & 4 & 4 & 5 \\
\hline 3 & 2 & 4 & 4 & 3 & 2 & 2 & 2 & 3 & 4 & 3 & 4 & 4 \\
\hline 3 & 3 & 1 & 4 & 4 & 3 & 2 & 3 & 4 & 4 & 3 & 4 & 4 \\
\hline 3 & 4 & 2 & 5 & 3 & 3 & 4 & 3 & 4 & 3 & 4 & 4 & 4 \\
\hline 4 & 1 & 4 & 5 & 5 & 2 & 4 & 3 & 4 & 4 & 4 & 4 & 4 \\
\hline 4 & 2 & 3 & 5 & 3 & 3 & 4 & 3 & 3 & 4 & 4 & 4 & 5 \\
\hline 4 & 3 & 2 & 5 & 4 & 3 & 4 & 2 & 5 & 4 & 4 & 5 & 5 \\
\hline 4 & 4 & 1 & 4 & 2 & 2 & 3 & 2 & 4 & 4 & 3 & 3 & 4 \\
\hline
\end{tabular}

$\begin{array}{cl}\text { SCORE } & \\ 5 & =\text { Excellent } \\ 4 & =\text { Good } \\ 3 & =\text { Enough } \\ 2 & =\text { Not Enough } \\ 1 & =\text { Less }\end{array}$




\section{Taguchi Design}

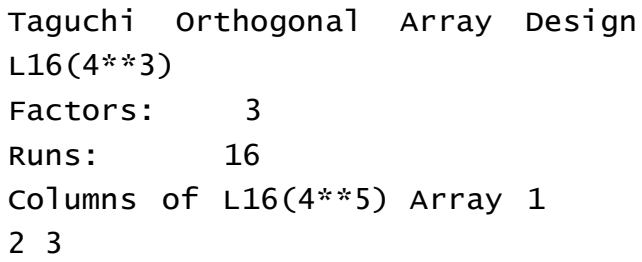

Taguchi Analysis: 1; 2; 3; ... versus INTELLECTUAL; EMOTIONAL CO; SOCIAL COMPE Response Table for Signal to Noise Ratios Nomina 1 is best $\left(10 * \log 10\left(\mathrm{Ybar}^{*} * 2 / \mathrm{s} * * 2\right)\right)$

\begin{tabular}{|c|c|c|c|}
\hline Leve1 & $\begin{array}{r}\text { INTELLECTUAL } \\
\text { COMPETENCY }\end{array}$ & $\begin{array}{r}\text { EMOTIONAL } \\
\text { COMPETENCY }\end{array}$ & $\begin{array}{l}\text { SOCIAL } \\
\text { COMPETE }\end{array}$ \\
\hline 1 & 15,34 & 13,20 & 13,54 \\
\hline 2 & 11,34 & 12,45 & 12,45 \\
\hline 3 & 13,16 & 13,21 & 13,13 \\
\hline 4 & 12,48 & 13,47 & 13,20 \\
\hline De1ta & 4,00 & 1,02 & 1,09 \\
\hline Rank & 1 & 3 & 2 \\
\hline \multicolumn{4}{|c|}{ Response } \\
\hline & INTELLECTUAL & EMOTIONAL & SOCIAL \\
\hline Leve1 & COMPETENCY & COMPETENCY & COMPETENCY \\
\hline 1 & 3,750 & 3,675 & 3,525 \\
\hline 2 & 3,250 & 3,500 & 3,575 \\
\hline 3 & 3,550 & 3,675 & 3,800 \\
\hline 4 & 3,725 & 3,425 & 3,375 \\
\hline De1ta & 0,500 & 0,250 & 0,425 \\
\hline Rank & 1 & 3 & 2 \\
\hline
\end{tabular}

Response Table for Standard Deviations

$\begin{array}{lrrr} & \text { INTELLECTUAL } & \text { EMOTIONAL } & \text { SOCIAL } \\ \text { Leve1 } & \text { COMPETENCY } & \text { COMPETENCY } & \text { COMPETENCY } \\ 1 & 0,6530 & 0,8094 & 0,7484 \\ 2 & 0,8793 & 0,8327 & 0,8529 \\ 3 & 0,7833 & 0,8071 & 0,8421 \\ 4 & 0,8836 & 0,7499 & 0,7558 \\ \text { De1ta } & 0,2307 & 0,0828 & 0,1045 \\ \text { Rank } & 1 & 3 & 2\end{array}$



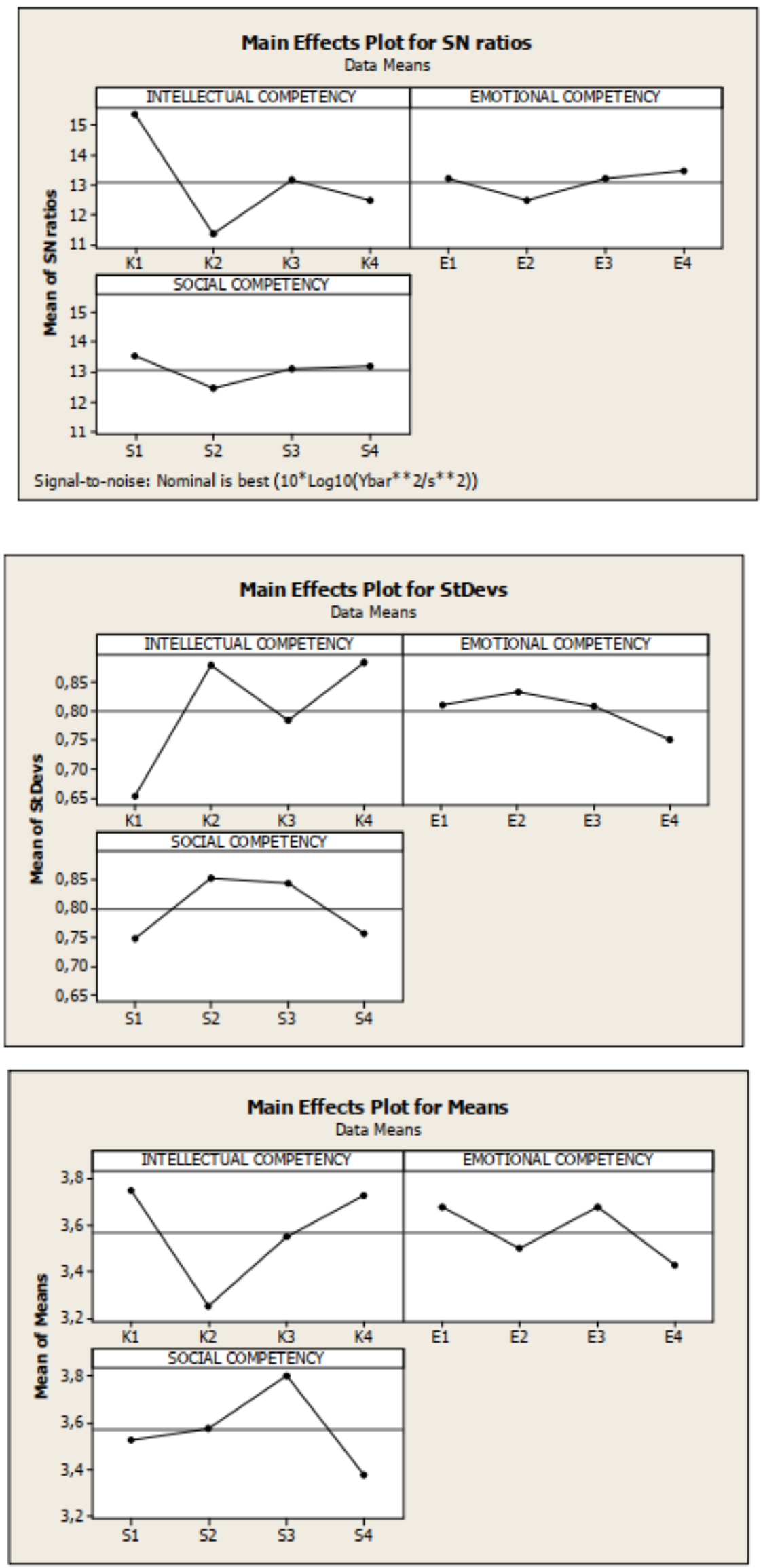

Fig. Taguchi Model 


\section{CONCLUSION}

Based on the Response Table for Signal to Noise Ratios Nominal is best, indicating that the three factors of intellectual, emotional, and social competence can be ranked by rank. Taguchi test results done got the value of the effect plot for means with the approach of table of means, then the intellectual competence is needed for the improvement of HR performance

\section{ACKNOWLEDGMENT}

\section{REFERENCES}

[1] Achmad, Zaenudin, 2009. Manajemen Sumber Daya Manusia, Cetakan Kedua, Penerbit Fajar, Jakarta.

[2] Anwar Prabu Mangkunegara, 2001. Manajemen Sumber Daya Manusia Perusahaan, Remaja Rosdakarya, Jakarta.

[3] Azwar, Saifudin, 2004, Reliabilitas dan Validitas, Cetakan V, Pustaka Pelajar, Yogyakarta.

[4] Cohen, L., 1995. Quality Function Deployment : How to Make QFD Work for you, Addison-Wesley Publishing Company, Massachusetts.

[5] F. Rahmadianto, 2018. Analisa Putaran Spindle dan Kedalaman Potong Terhadap Keausan Pahat Positive dan Negative Rhombic Insert, Institut Teknologi Nasional, Malang.

[6] F. Rahmadianto, 2014. Upaya Peningkatan Sifat Mekanik Baja Mild Steel Melalui Perbaikan Kualitas dengan Heat Treatment Annealing dan Holding Time pada Heat Treatment dengan Taguchi Method, Universitas Brawijaya, Malang.

[7] Moeheriono. 2009. Pngukuran Kinerja Berbasis Kompetensi. Bogor : Ghalia, Indonesia.

[8] Prawirosentono suyadi, 1999. Kebijakan Kinerja Karyawan. Yogyakarta : BPFE.

[9] Sugiyono, 2004. Metode Penelitian Bisnis, Cetakan Ketujuh, Alfabeta, Bandung

[10] Tjiptono,F. dan Diana, A., 2002. Total Quality Management, Edisi keempat Cetakan 2, Andy, Yogyakarta. 